\title{
Thanks to all those who reviewed for Systematic Reviews in 2014
}

David Moher ${ }^{1 *}$, Paul Shekelle ${ }^{2}$ and Lesley A Stewart ${ }^{3}$

\section{Contributing reviewers}

A peer-reviewed journal would not survive without the generous time and insightful comments of the reviewers, whose efforts often go unrecognized. Although final decisions are always editorial, they are greatly facilitated by the deeper technical knowledge, scientific insights, understanding of social consequences, and passion that reviewers bring to our deliberations. For these reasons, the Editors-in-Chief and staff of the journal warmly thank the 219 reviewers whose comments helped to shape Systematic Reviews, for their invaluable assistance with review of manuscripts for the journal in Volume 3 (2014).

Jawdat Abdulla

Denmark

Clive E. Adams

UK

Myzoon Ali

UK

Eitan Amir

Canada

Raghupathy Anchala

India

Lisa Askie

Australia

Marc Avey

Canada

Ethan Balk

USA

Joel Bamford

USA

David Bellinger

USA

Jennifer Bellis

UK
Kate Bennett

UK

Nancy Berkman

USA

Jesse Berlin

USA

Laurent Beydon

France

Unnikrishnan Bhaskaran

India

Zhaoxiang Bian

Hong Kong

John Billi

USA

Giuseppe Biondi-Zoccai

Italy

Jan Blaakaer

Denmark

Stefania Boccia

Italy

Laura Bonnett

UK
Jonathan Boote

UK

Andrew Booth

UK

Isabelle Boutron

France

Caroline Braet

Belgium

Thomas Butt

UK

Deborah Caldwell

UK

Alun Cameron

Australia

Chris Cameron

Canada

Fiona Campbell

UK

Kristin Carson

Australia

Adriana Castelli

UK

* Correspondence: dmoher@ohri.ca

${ }^{1}$ Ottawa Hospital Research Institute and University of Ottawa, Ottawa, Canada

${ }^{2}$ Southern California Evidence-based Practice Center, Santa Monica, CA, USA

${ }^{3}$ Centre for Reviews and Dissemination, University of York, York, UK 
Ferrán Catalá-López

Spain

Yaping Chang

Canada

Deepak Chawla

India

Hui-Ching Chuang

Taiwan

Americo Cicchetti

Italy

Shannon Cope

Canada

Alessandro Coppo

Italy

Miranda Cumpston

Australia

Huw Davies

UK

Cinzia del Giovane

Italy

Vakaramoko Diaby

USA

Sofia Dias

UK

Kelly Dickson

UK

Therese Dowswell

UK

Janine Dretzke

UK

Isabelle Durand-Zaleski

France

Kerry Dwan

UK

Donald Edmondson

USA

Regina El Dib

Brazil

Alexandra Ellis

USA

Louise Falzon

USA

Forough Farrokhyar

Canada
Katalin Fekete

Hungary

Jonas David Finger

Germany

Alan Fleischer

USA

Kate Flemming

UK

Rosanne Freak-Poli

Australia

Tomohiro Funakoshi

USA

James Galipeau

Canada

Rebecca Ganann

Canada

Ruth Garside

UK

Gerald Gartlehner

Austria

Charlotte Gaydos

USA

Klaus Gebel

Australia

Su Golder

UK

Sean Grant

UK

Katja Grasic

UK

Janette Greenhalgh

UK

Trisha Greenhalgh

UK

Kelly Grindrod

Canada

Ulrich Grouven

Germany

Annetje Guedon

Netherlands

Ruth Gwernan-Jones

UK

Susanne Hempel

USA
William Hersh

USA

Renee Hessian

Canada

Suzanne Hill

Australia

Caroline Homer

Australia

Min Kyung Hyun

South Korea

Justin Jagosh

Canada

Nikki Jahnke

UK

Heethal Jaiprakash

Malaysia

Anthony James

UK

Wayne Jonas

USA

Cassandra Josephson

USA

Robert Kane

USA

Marloes Kleinjan

Netherlands

Levente Kriston

Germany

Oliver Kuss

Germany

William Lambert

USA

Toby Lasserson

UK

Mariska M. G. Leeflang

Netherlands

Anne Lethaby

New Zealand

James Lewis

USA

Yuan Li

China

Tianjing Li

USA 
Kuancho Liao

Taiwan

Helen Liley

Australia

Yu-Tsai Lin

Taiwan

Kathleen Lohr

USA

Yoon Kong Loke

UK

Frank Louws

USA

Georgios Lyratzopoulos

UK

Laxmaiah Manchikanti

USA

Ana Marusic

Croatia

Annette Matthews

USA

Alain Mayhew

Canada

Evan Mayo-Wilson

USA

Lawrence Mbuagbaw

Cameroon

Catriona McDaid

UK

Marian McDonagh

USA

G. J. Melendez-Torres

UK

Virginia Minogue

UK

Suneeta Mittal

India

Kaelan Moat

Canada

David Moher

Canada

Ali Montazeri

Iran

Horieh Moosavi

Iran
Manuel Muñoz

Spain

Michael Murphy

UK

Huseyin Naci

UK

Sreekumaran Nair

India

Suma Nair

India

Sydne Newberry

USA

Mark Newman

UK

Lisbeth Nilas

Denmark

Sarah Nolan

UK

Susan L. Norris

USA

Allison Ober

USA

Stephen O'Leary

Australia

Wendy Olsen

UK

Maya O'Neil

USA

Michael Ostacher

USA

Carrie Patnode

USA

Porjai Pattanittum

Thailand

Mark Pearson

UK

Einat Peles

Israel

Bob Phillips

UK

Julie Polisena

Canada

Jennie Popay

UK
Stephanie Prince

Canada

Marsha Raebel

USA

Rajeev Ramchand

USA

Andrew Rankin

UK

H Fisher Raymond

USA

Barnaby Reeves

UK

Toby Richards

UK

David Riley

USA

Emilie Robert

Canada

Mark Rodgers

UK

Martin Rohling

USA

Maroeska Rovers

Netherlands

Gerta Rücker

Germany

George Rutherford

USA

Laura Sampietro-Colom

Spain

Margaret Sampson

Canada

Connie Schardt

USA

Roberta Scherer

USA

David Schriger

USA

Jodi Segal

USA

Shelley Selph

USA

Tatyana Shamliyan

USA 
Larissa Shamseer

Canada

Beverley Shea

Canada

Paul Shekelle

USA

Nathan Shippee

USA

Vijay Shukla

Canada

Jean Sibilia

France

Becky Skidmore

Canada

Rebecca Smyth

UK

Andy Spencer

UK

Ann Sprague

Canada

Robert Stanton

Australia

Adrienne Stevens

Canada

Kitty Stewart

UK

Lesley Stewart

UK

Catalina Suarez-Cuervo

USA

Carolyn Summerbell

UK

Yemisi Takwoingi

UK

Howard Thom

UK
Carl Thompson

UK

Hilary Thomson

UK

Judith Thornton

UK

Jin-Hui Tian

China

Guy Tsafnat

Australia

Tony Tse

USA

Alexander Tsertsvadze

Canada

Ioannis Tzanakis

Greece

Jelle van Cauwenberg

Belgium

Wynanda van Enst

Netherlands

Gert van Valkenhoef

Netherlands

Andre Veras

Brazil

Areti Angeliki Veroniki

Canada

Liat Vidal

Israel

Jean-Louis Vincent

Belgium

John Wahren

Sweden

Byron Wallace

USA

Rebecca Waller

USA
Debbie Warman

USA

Vivian Welch

Canada

Nicky Welton

UK

Suzanne West

USA

Gill Westhorp

Australia

Marie Elaine Westwood

UK

Penny Whiting

UK

Evelyn Whitlock

USA

Craig Whittington

UK

Kumanan Wilson

Canada

Paul M. Wilson

UK

Michael Wilson

Canada

Nicole Wolfe

USA

Geoff Wong

UK

Judy Wright

UK

Kath Wright

UK

Po-Yin Yen

USA

Junhua Zhang

China 